\title{
Two water waves in subsurface interaction on a shear flow
}

\author{
Peder A. Tyvand
}

Received: 18 April 2021 / Accepted: 22 September 2021 / Published online: 5 February 2022

(C) The Author(s) 2022

\begin{abstract}
Two plane-traveling water waves are superposed, with a background shear flow. The horizontal shear flow varies exponentially, with infinite depth. The present idea is to satisfy the two-dimensional vorticity equation for the second-order subsurface interaction between the two waves. The free-surface conditions are linearized, while the remaining (subsurface) nonlinearity has its amplitude set by the product of the two wave amplitudes. The first traveling wave is assumed steady, satisfying the same Helmholtz equation as the shear flow. Thereby, one single frequency enters the model, dictated by the second traveling wave. There are in total four wave components generated by subsurface interactions. Two of these are rotational waves arising from the exact quadratic interactions between the two first-order waves. The two other waves are compensational irrotational waves setup by linearized free-surface conditions, since the rotational waves alone are unable to satisfy these conditions.
\end{abstract}

Keywords Fourier components · Shear flow · Vorticity $\cdot$ Water waves

\section{Introduction}

The classical modeling of water waves is based on potential theory, but in the presence of a basic shear flow, this is not an option. The only exception is the case of uniform vorticity, where the perturbation velocity remains a potential flow, with changed nonlinear boundary conditions in the general modeling. Simmen and Saffman [1] performed deep-water computations of steady periodic nonlinear water waves with uniform vorticity. Teles da Silva and Peregrine [2] performed such computations for finite depth, and computations with high accuracy have recently been performed by Hur and Wheeler [3].

It is challenging to take non-uniform vorticity into account for finite-amplitude water waves. The paper by Dalrymple [4] was based on the standard Eulerian description of motion. Much earlier, an exceptional class of such steady waves was established in the literature. These are the Gerstner waves [5], described by Lamb [6]. The representation of Gerstner waves as strongly nonlinear steady waves rests on the Lagrangian description. Fully nonlinear Gerstner waves seem not to have been satisfactorily translated into the Eulerian description, where the confrontation with the steady vorticity equation can be made explicit.

P. A. Tyvand $(\varangle)$

Faculty of Mathematical Sciences and Technology, Norwegian University of Life Sciences, 1432 Ås, Norway

e-mail: peder.tyvand@nmbu.no 
In the present paper, we will focus on satisfying the vorticity equation exactly since this is downplayed in the literature on nonlinear water waves with variable vorticity. Nwogu [7] incorporated the exact time-dependent vorticity equation in his work, but during his study, he made simplifications that ignored the redistribution of vorticity in the vertical direction. Several other authors have studied finite-amplitude waves with non-uniform vorticity under the assumption that the flow is steady. Thereby, the time-dependent vorticity equation is no longer an issue, being replaced by a general nonlinear functional relationship between the vorticity and the streamfunction. Such approaches to finite-amplitude waves have been presented by Constantin et al. [8], Ko and Strauss [9], and Cheng et al. [10].

Exact analytical work on the nonlinear vorticity equation seems to be lacking in the literature on water waves, as a contrast to the efforts to satisfy the nonlinear free-surface conditions. Our present work will, therefore, focus on the subsurface vortex interactions in the bulk fluid, while we will keep the free-surface conditions linearized. Analytical knowledge on nonlinear interactions between a free surface and continuous vortex distributions is limited, but such approaches are naturally overshadowed by the complex physics of shear flow instabilities and critical layers. The work by Milewski and Benney [11] is relevant for higher-order interactions that our model will involve beyond the presented second-order theory.

Much numerical work has been published concerning discrete vortices as well as continuous vortices submerged below a free surface. A broad overview of nonlinear vortex interactions with a free surface was given in the review articles by Peregrine [12] and Sarpkaya [13]. Discrete vortices in free subsurface motion [14,15] can have strong nonlinear effects, which may lead to surface breaking.

Concerning water waves, nonlinearities at the free surface have been investigated more closely than the nonlinearities due to finite motions within the fluid. From a physical point of view, this is a paradox because the nonlinearities from submerged objects in finite-amplitude motion close to the surface easily become stronger than the free-surface nonlinearities. An interesting paper was written by $\mathrm{Wu}[16]$, illustrating the importance of finite motions of objects below the free surface, illustrating how they may overshadow the nonlinearities at the free surface. A lot of work remains to be done concerning nonlinearities in the fluid itself, which are of two different categories: (i) Vortex interactions with or without influence from fixed solid boundaries and (ii) Finite motions of submerged objects with neglect of vorticity.

These two categories of subsurface nonlinearities are often inseparable from one another. Vortex shedding is such a mixed phenomenon, which is very difficult to capture in any type of modeling from first principles. A dimensionless number to characterize this phenomenon is the Keulegan-Carpenter number, but its application is mainly linked to observations [17]. The theoretical models can usually not be closed without the use of empirical constants, with the Morison equation as a standard example. Only rudimentary theories exist concerning the influence of the Keulegan-Carpenter number on inviscid vortex flows, even though this number itself has no explicit link to viscosity and should be applicable to inviscid flows.

As pointed out by Tyvand [18], it is not common to pose a nonlinear water-wave problem as a causal CauchyPoisson problem. A customary starting point is a linearized periodic wave in space and time, where causality is not an issue. In this context, nonlinearity seems to come naturally in terms of an amplitude expansion, still without a causal first-principle point of view. An important substitute for physical causality is the mathematical stability analysis. However, the family of physically admissible perturbations in an inviscid fluid is then constrained by Lord Kelvin's circulation theorem, which essentially prevents the generation of vorticity. A nonlinear initial value problem circumvents these constraints since it is not necessary to argue for the causation of a mathematical initial value problem. Nonlinear initial value problems offer legal ways to circumvent the strict physical constraints for vorticity generation in inviscid flow models. This approach is admittedly idealized but not artificial for capturing natural viscous processes with a long time scale since their further macroscopic evolution may be essentially inviscid at short time scales.

In the present paper, we will consider subsurface vortex interactions. A basic shear flow is assumed, supporting rotational modes of surface waves, which serve as parental wave modes that combine to offspring wave modes with perturbation vorticity. We will consider two linearized rotational wave modes and calculate their rotational offspring wave generated from their subsurface interaction. 
A subsurface rotational flow and an initiation of surface motion are two concerns that are difficult to combine in a consistent nonlinear initial value problem. It appears that subsurface nonlinear interaction of rotational flows tends to be of a different nature compared with the surface flows, even within linearized theory for free-surface displacements. Mismatches between nonlinear subsurface vortex interactions and their influence on the free surface tend to induce compensatory potential flows for the free-surface conditions to be satisfied. These constraints make it hard to establish any steady nonlinear rotational water waves beyond the validity of a second-order amplitude expansion.

Hydrodynamic stability is an important issue for shear flows. Stability is never an issue for linearized water waves because it has no meaning to perturb a wave according to linear theory. The question is, therefore, whether a linearized surface wave exists with purely oscillatory time dependence. We will take the simplest version of such waves and study their subsurface interaction according to the vorticity equation. This interaction will be exact to second order in the amplitudes of the two interacting waves.

\section{The fully nonlinear mathematical model}

We consider an inviscid and incompressible fluid (liquid) in the gravity field, where the undisturbed free surface is aligned along a horizontal $x$ axis. We will consider free two-dimensional gravitational waves with surface elevation $\eta(x, t)$. The free surface is subject to constant pressure. Surface tension is neglected. Cartesian coordinates $x, y$ are introduced, where the $y$-axis is directed upwards in the gravity field. The gravitational acceleration is $g$, and $\rho$ denotes the constant fluid density. The velocity vector components $(u, v)$ are represented by the streamfunction $\Psi(x, y, t)$ as follows:

$u=\frac{\partial \Psi}{\partial y}, \quad v=-\frac{\partial \Psi}{\partial x}$,

which by definition satisfies mass balance for incompressible flow. The evolution equation for the streamfunction is the vorticity equation:

$\frac{\partial\left(\nabla^{2} \Psi\right)}{\partial t}+\left(\frac{\partial \Psi}{\partial y} \frac{\partial}{\partial x}-\frac{\partial \Psi}{\partial x} \frac{\partial}{\partial y}\right) \nabla^{2} \Psi=0$.

It expresses that the vorticity $-\nabla^{2} \Psi$ of an individual fluid particle is conserved. It also shows the curl of the acceleration field is zero, which follows from Euler's equation of motion

$\vec{a}=-\frac{1}{\rho} \nabla p-g \vec{j}$,

where $\vec{a}$ is the acceleration field and $\vec{j}$ is the vertical unit vector. The pressure is denoted by $p$. The vorticity is $-\nabla^{2} \psi$.

The nonlinear kinematic free-surface condition is

$\frac{\partial \eta}{\partial t}+\frac{\partial \Psi}{\partial y} \frac{\partial \eta}{\partial x}+\frac{\partial \Psi}{\partial x}=0, \quad y=\eta(x, t)$.

The nonlinear dynamic boundary condition is given by the tangential component of the Euler equation along the free surface. It can be written as follows:

$\vec{a} \cdot \vec{\tau}=-g \vec{j} \cdot \vec{\tau}, \quad y=\eta(x, t)$,

following from the vanishing tangential derivative of the pressure along the free surface. Here, $\vec{\tau}$ is a tangential vector along the free surface

$\vec{\tau}=\vec{i}+\frac{\partial \eta}{\partial x} \vec{j}$. 
The nonlinear dynamic condition (5) written in terms of the functions $\psi(x, y, t)$ and $\eta(x, t)$ is

$$
\begin{aligned}
& \frac{\partial^{2} \Psi}{\partial y \partial t}-\frac{\partial \eta}{\partial x} \frac{\partial^{2} \Psi}{\partial x \partial t}+\frac{\partial \Psi}{\partial y} \frac{\partial^{2} \Psi}{\partial x \partial y}-\frac{\partial \Psi}{\partial x} \frac{\partial^{2} \Psi}{\partial y^{2}} \\
& -\frac{\partial \eta}{\partial x}\left(\frac{\partial \Psi}{\partial y} \frac{\partial^{2} \Psi}{\partial x^{2}}-\frac{\partial \Psi}{\partial x} \frac{\partial^{2} \Psi}{\partial x \partial y}\right)=-g \frac{\partial \eta}{\partial x}, \quad y=\eta(x, t),
\end{aligned}
$$

In the present paper, we will only consider infinite depth, where the wave field decays as $y \rightarrow-\infty$.

We have now developed a mathematical model for two-dimensional water waves with a general velocity field. The formulation can be completed by posing an initial value problem with the initial distributions of the functions $\psi(x, y, 0)$ and $\eta(x .0)$ given. By considering this inviscid flow evolution as a mathematical initial value problem, we do not need to take the causality behind the vorticity field into account.

\section{Linearized surface waves}

We will first look at the general case of 2D linearized surface gravity waves on a unidirectional shear current $U(y)$, which gives the total streamfunction:

$\Psi=F(y)+\psi(x, y, t)$,

where the wave field is represented by the streamfunction perturbation $\psi$. The function $F(y)$ is specifies the basic flow distribution $U(y)$ by the definition:

$F^{\prime}(y)=U(y)$,

whereby $F(y)$ is the antiderivative of $U(y) . \Psi(y)$ is a separate streamfunction for the basic flow. The surface wave is represented by the perturbation $\psi(x, y, t)$.

The nonlinear vorticity equation (2) can be expressed as follows:

$\frac{\partial\left(\nabla^{2} \psi\right)}{\partial t}=-U(y) \frac{\partial\left(\nabla^{2} \psi\right)}{\partial x}+\frac{\partial \psi}{\partial x}\left(U^{\prime \prime}(y)+\frac{\partial\left(\nabla^{2} \psi\right)}{\partial y}\right)-\frac{\partial \psi}{\partial y} \frac{\partial\left(\nabla^{2} \psi\right)}{\partial x}$,

and its linearized version is

$\frac{\partial\left(\nabla^{2} \psi\right)}{\partial t}=-U(y) \frac{\partial\left(\nabla^{2} \psi\right)}{\partial x}+U^{\prime \prime}(y) \frac{\partial \psi}{\partial x}$,

to be satisfied for linearized water waves. The linearized version of the kinematic free-surface condition (4) is

$\frac{\partial \eta}{\partial t}+U(0) \frac{\partial \eta}{\partial x}=-\left.\frac{\partial \psi}{\partial x}\right|_{y=0}$,

while the dynamic free-surface condition (7) reduces to

$\frac{\partial^{2} \psi}{\partial y \partial t}+U(0) \frac{\partial^{2} \psi}{\partial x \partial y}-U^{\prime}(0) \frac{\partial \psi}{\partial x}=-g \frac{\partial \eta}{\partial x}, \quad y=0$,

in its general linearized version.

The linearized version of Euler's equation of motion (3) has the components:

$$
\begin{aligned}
& \frac{\partial^{2} \psi}{\partial y \partial t}+U(y) \frac{\partial^{2} \psi}{\partial x \partial y}-U^{\prime}(y) \frac{\partial \psi}{\partial x}=-\frac{1}{\rho} \frac{\partial p}{\partial x}, \\
& \frac{\partial^{2} \psi}{\partial x \partial t}+U(y) \frac{\partial^{2} \psi}{\partial x^{2}}=\frac{1}{\rho} \frac{\partial p}{\partial y}+g .
\end{aligned}
$$




\subsection{Oscillatory linearized waves}

We have now formulated the linearized time-dependent problem for surface waves on a shear flow $U(y)$. A general Fourier component solution is

$\psi=Y(y) \mathrm{e}^{\mathrm{i}(k x-\omega t)}$,

which we insert in the linearized vorticity equation (11). $k$ is the wavenumber and $\omega$ is the angular frequency. This leads to the standard inviscid Orr-Sommerfeld equation (also called the Rayleigh equation):

$\frac{Y^{\prime \prime}(y)}{Y(y)}-k^{2}=\frac{U^{\prime \prime}(y)}{U(y)-\omega / k}$

see e.g., Miles [19]. The surface elevation accompanying the streamfunction (16) is expressed as follows:

$\eta(x, t)=\eta_{0} \mathrm{e}^{\mathrm{i}(k x-\omega t)}$,

where we introduce the elevation amplitude $\eta_{0}$. It is related to the streamfunction amplitude $Y(0)$ as follows:

$\eta_{0}=\frac{Y(0)}{\omega / k-U(0)}$

according to the kinematic condition (12).

The governing equation (17) has to be solved in order to determine the dispersion relation by inserting the solution in the linearized dynamic free-surface condition (13), which reduces to

$\frac{Y^{\prime}(0)}{Y(0)}=\frac{U^{\prime}(0)}{U(0)-\omega / k}+\frac{g}{(U(0)-\omega / k)^{2}}$.

In the Orr-Sommerfeld equation, a well-known singularity appears if there is a level where the shear flow velocity coincides with the phase speed $\omega / k$. In the special case where the singularity is located at the linearized free surface $y=0$, the singularity will also appear in the free-surface conditions.

We have now formulated the oscillatory flow problem for linearized water waves on a shear flow. In the present context, we will not attempt to solve it, since we will focus on steady waves.

\subsection{Derivation of steady-linearized waves}

As pointed out by Peregrine [12], the most general class of steady nonlinear waves with vorticity satisfies the condition:

$\nabla^{2} \psi=f(\psi)$,

expressing that the vorticity is constant along each individual steady streamline where $\psi=$ constant. Ko and Strauss [9] explored this relationship numerically for finite-amplitude waves with variable vorticity.

We will linearize the free-surface conditions and only consider subsurface nonlinear effects in this paper. No we go into more detail with the steady-linearized free-surface flow where $\omega=0$. The Orr-Sommerfeld equation (17) takes the much simpler form:

$\frac{Y^{\prime \prime}(y)}{Y(y)}-k^{2}=\frac{U^{\prime \prime}(y)}{U(y)}$,

which is without singularities, assuming $U(y) \neq 0$ for all $y \leq 0$ so that $U(y)$ has no subsurface sign change. The most general solution of Eq. (22) is given by

$\frac{Y^{\prime \prime}(y)}{Y(y)}-k^{2}=\frac{U^{\prime \prime}(y)}{U(y)}=G(y)$,

where $G(y)$ is an arbitrary function. The eigenvalue problem for $Y(y)$ will then become

$Y^{\prime \prime}(y)-k^{2} Y(y)=G(y) Y(y)$. 
Requiring steady flow, the function $G(y)$ is constrained by the general nonlinear relationship (21) for the streamfunction $\psi(x, y)$, which in its linearized version is

$\nabla^{2} \psi-\beta^{2} \psi=0$,

ensuring that the vorticity is constant along a steady streamline. Here we have excluded the well-established case of uniform vorticity where $f(\psi)=$ constant. We insert this relationship in the steady-linearized vorticity equation (11) to obtain

$U^{\prime \prime}(y)=\beta^{2} U(y)$,

after canceling the time derivative. Thereby, the shear flow is constrained to have an exponential variation with height

$U(y)=U_{0} \mathrm{e}^{\beta y}$,

satisfying $U(-\infty)=0$. Here, we have introduced the notation $U(0)=U_{0}$. We see that the shear flow itself has to satisfy a Helmholtz type of equation (25). We note the sign change in this equation compared with the conventional Helmholtz equation, which is necessary to obtain normal modes that decay exponentially in a semi-infinite domain. The vertical dependency for the streamfunction is

$q=\sqrt{\beta^{2}+k^{2}}$,

according to Eq. (23). We see that the function $G(y)$ is constant $\left(G(y)=\beta^{2}\right)$, and the steady solution for the streamfunction of the propagating wave is

$\psi(x, y)=A_{1} \mathrm{e}^{\mathrm{i} k x+q y}$,

where the amplitude $A_{1}$ is arbitrary in linear theory. We rewrite this steady streamfunction in real form with a phase shift angle $\theta$

$\psi(x, y)=A_{1} \mathrm{e}^{q y} \cos (k x-\theta)=A_{1} \mathrm{e}^{\sqrt{\beta^{2}+k^{2}} y} \cos (k x-\theta)$,

and by doing that, we avoid the introduction of a phase shift angle for the time-dependent second wave that we will introduce below, represented by a streamfunction $\tilde{\psi}(x, y, t)$.

The spatial decay rate for the wave field follows from Eq. (20)

$q=\beta+\frac{g}{U_{0}^{2}}$,

and it shows that the spatial decay of the wave is stronger than the decay of the shear flow. The shear flow effect dominates the spatial decay of the steady wave when $\beta U_{0}^{2}>g$. When $\beta U_{0}^{2}<g$, the gravitational influence at the free surface is the main cause for the spatial decay of the steady wave. Inserting $\beta=\sqrt{q^{2}-k^{2}}$, another formula for $q$ is derived

$q=\frac{1}{2}\left(\frac{g}{U_{0}^{2}}+\frac{\left(k U_{0}\right)^{2}}{g}\right)$.

Equating these two expressions for $q$ gives a dispersion relation for steady waves

$k=\frac{g}{U_{0}^{2}} \sqrt{1+2 \frac{\beta U_{0}^{2}}{g}}=\frac{g}{U_{0}^{2}}+\beta+O\left(\beta^{2}\right)$.

Here, we show how the wavenumber $k$ depends on the shear flow, and we include an expansion for small shear parameter $\beta \geq 0$. For a given basic flow $U(y)=U_{0} \exp (\beta y)$, there is only one wavenumber $k$ that gives steadylinearized waves, in the coordinate system where the fluid is at rest at infinite depth. The classical deep-water dispersion relation $k=g / U_{0}^{2}$ emerges in the limit $\beta \rightarrow 0$, with $U_{0}$ representing the phase velocity of steady waves on a uniform flow. 
Fig. 1 Definition sketch for the shear flow $U(y)$ and the steady wave elevation $\eta(x)$

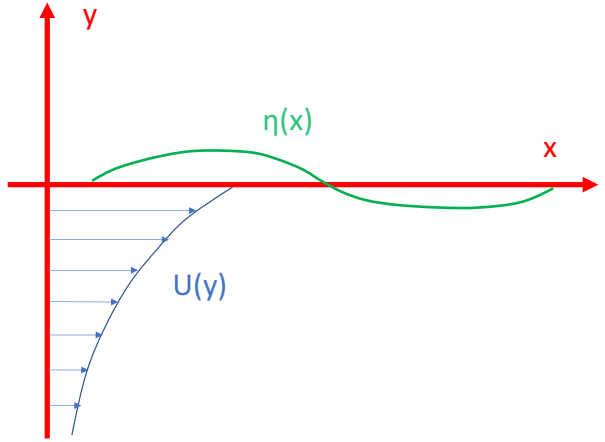

We rewrite the dispersion relation (33) for steady waves in dimensionless form:

$\frac{U_{0}^{2}}{g} k=\sqrt{1+2 \frac{\beta U_{0}^{2}}{g}}$.

This formula shows how the shear gives a shorter steady wave than a uniform flow $U_{0}$.

It is advantageous that the steady wave and the shear flow itself satisfy the same Helmholtz-type equation:

$\nabla^{2} \psi=\beta^{2} \psi$,

because there cannot be any vortex interactions between two sets of normal-mode eigenfunctions of the same Helmholtz-type equation. This is easily proven by inserting the sum of two eigenfunctions $\psi_{1}$ and $\psi_{2}$ into the nonlinear vorticity equation: (2). $\Psi=\psi_{1}+\psi_{2}$ gives

$\frac{\partial \omega}{\partial t}=\beta\left(\frac{\partial \psi_{1}}{\partial y} \frac{\partial \psi_{2}}{\partial x}-\frac{\partial \psi_{1}}{\partial x} \frac{\partial \psi_{2}}{\partial y}+\frac{\partial \psi_{2}}{\partial y} \frac{\partial \psi_{1}}{\partial x}-\frac{\partial \psi_{2}}{\partial x} \frac{\partial \psi_{1}}{\partial y}\right)=0$,

where the proportionality between each streamfunction and its vorticity (Eq. (35)) has been applied to show that the induced vorticity from the two combined rotational flows is identially zero.

When two rotational flows that are superposed do not induce any vorticity, they have no interaction at all. The absence of subsurface interactions between an exponentially varying shear flow and its supported steady surface wave means that there is neither instability [20] nor critical layer [21] induced by this legal steady wave. Figure 1 gives a definition sketch for the shear flow and its accompanying steady wave, which is uniquely given apart from its amplitude and phase parameter.

\section{An additional traveling wave}

We will add another wave component to the steady-linearized system that we have established, where the shear flow is given by Eq. (27). A distinct second wave must be time dependent, with a streamfunction $\tilde{\psi}(x, y, t)$ generally defined as follows:

$\tilde{\psi}=Z(y) \mathrm{e}^{\mathrm{i}(\kappa x-\omega t)}$,

according to the above formula (16). The above equation (20) is still valid, rewritten with this modified notation as follows:

$\frac{Z^{\prime}(0)}{Z(0)}=\frac{\beta U_{0}}{U_{0}-\omega / \kappa}+\frac{g}{\left(U_{0}-\omega / \kappa\right)^{2}}$.

The solution for the streamfunction is then

$\tilde{\psi}=A_{2} \mathrm{e}^{\alpha y} \mathrm{e}^{\mathrm{i}(\kappa x-\omega t)}$, 


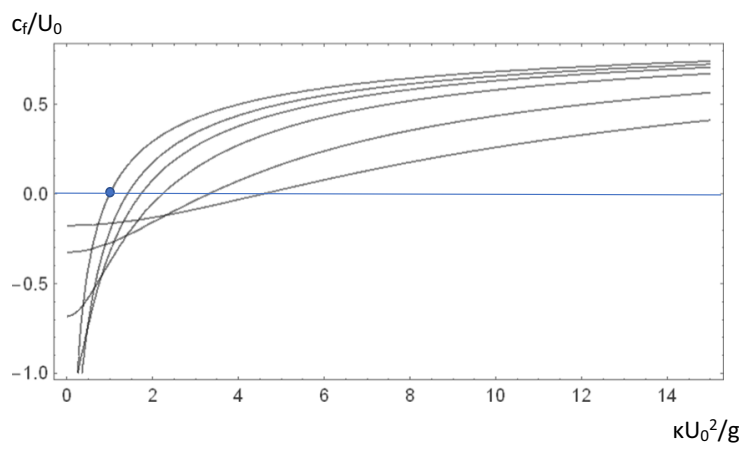

Fig. 2 Numerical evaluation of the dispersion relation for a time-dependent wave. The dimensionless phase velocity $\mathrm{c}_{\mathrm{f}} / \mathrm{U}_{0}$ is plotted as a function of the dimensionless wave number $\mathbf{k U}_{0}{ }^{2} / \mathbf{g}$ for increasing values of the shear flow decay parameter $\beta \mathrm{U}_{0}^{2} / \mathrm{g}=0,0.5,1,2,5$, 10. The wave numbers for steady waves are represented by the points where $c_{f}=0$. These wave numbers increase with increasing shear flow parameter $\beta$. A blue circle shows the smallest of these wave numbers for steady waves. This is $\mathbf{k}=\mathrm{g} / \mathbf{U}_{0}{ }^{2}$ representing uniform flow $(\beta=0)$, where $c_{\mathrm{f}} / \mathbf{U}_{0}=\mathbf{1}$ by definition

where the spatial decay rate is

$\alpha=\frac{\beta U_{0}}{U_{0}-\omega / \kappa}+\frac{g}{\left(U_{0}-\omega / \kappa\right)^{2}}$.

Admittedly, the unsteady wave may in general admit instabilities as well as critical layer, if we allow a general complex time dependence. Since the free surface is linearized, the wave itself cannot be unstable as long as its purely oscillatory behavior is legal in terms of mathematical consistency. In the present paper, we look exclusively at the vortex interaction of this second oscillatory wave with the well-behaved steady surface wave that we introduced above. The Orr-Sommerfeld equation (17) is evaluated at the linearized free surface $y=0$ to give

$\alpha^{2}=\frac{\beta^{2} U_{0}}{U_{0}-\omega / \kappa}+\kappa^{2}$,

We introduce the auxiliary function $\xi(\kappa, \omega)$ defined by

$\xi=U_{0}-\frac{\omega}{\kappa}$,

which measures the phase velocity in a coordinate system where the undisturbed free surface is at rest. Thereby, we can rewrite the above formulas (40) and (41) compactly as

$$
\begin{aligned}
\alpha \xi & =\beta U_{0}+\frac{g}{\xi}, \\
\alpha^{2} \xi^{2} & =\beta^{2} U_{0} \xi+\kappa^{2} \xi^{2},
\end{aligned}
$$

and by elimiating $\alpha \xi$, we get the implicit dispersion relation

$\beta^{2} U_{0} \xi+\kappa^{2} \xi^{2}=\left(\beta U_{0}+\frac{g}{\xi}\right)^{2}$,

which we will study numerically. In principle, the dispersion relation expresses the dimensionless angular frequency $\omega g / U_{0}^{2}$ as a function of the dimensionless wave number $\kappa g / U_{0}$ and the dimensionless shear parameter $\beta U_{0}^{2} / g$. In Figure 2, we will plot the dimensionless phase velocity $c_{f} / U_{0}$ as a function of the wave number and the shear parameter.

One may of course, make a Galilean transformation so that this time-dependent wave again becomes steady, but then a nonzero flow at infinite depth must be allowed. We have, thus, been stricter than necessary from a mathematical point of view with our definition of steady waves, but there are some advantages with this approach. The exponential distribution for the basic flow stands out as an eigenfunction of the Helmholtz equation. This underlying shear flow dictates the behavior of the steady water wave, which is a normal-mode solution of the same Helmholtz equation that is obeyed by the shear flow. 


\subsection{Phase and group velocities}

Energy flux on a shear flow is a difficult topic, referring to Brevik and Sollie [22]. We will not give any details other than calculating the group velocity for the general case of time-dependent traveling waves. The phase velocity of this time-dependent wave is

$c_{f}=\frac{\omega}{\kappa}$,

measured relative to the stagnant fluid at infinite depth. The phase velocity is prescribed by the implicit dispersion relation (45). The case of a steady wave that we have treated above, emerges as the special case $c_{f}=0$, which means physically that a downstream wave is convected by the shear flow in the opposite direction so that the surface waves appears to be non-propagating. However, there is a nonzero group velocity even in the case of a steady wave.

We will now calculate the group velocity generally defined as follows:

$c_{g}=\frac{\mathrm{d} \omega}{\mathrm{d} \kappa}$,

which is preferably done in terms of dimensionless variables. A quick way of introducing dimensionless formulas is by putting $g=1$ and $U_{0}=1$. Thereby, we introduce the gravitational length unit $U_{0}^{2} / g$, the undisturbed surface flow $U_{0}$ is the velocity unit, while the time unit is $U_{0} / g$. The dimensionless version of the implicit dispersion relation (45) is

$\kappa^{2} \xi^{2}+\beta^{2} \xi-\beta^{2}-2 \frac{\beta}{\xi}-\frac{1}{\xi^{2}}=0$,

to be studied numerically. We then apply the auxiliary variable $\xi(\kappa, \omega)$ which was defined by Eq. (42) above. Its dimensionless version is

$\xi=1-\frac{\omega}{\kappa}=1-c_{f}$.

After inserting Eq. (49) in Eq. (48), we evaluate the dimensionless dispersion relation $c_{f}(\kappa, \beta)$ numerically. It is plotted in Figure 2. The transformations back from the dimensionless quantities in the text to the quantities with dimension in the figure captions are as follows:

$\left(c_{f}, c_{g}, \kappa, \beta\right) \rightarrow\left(\frac{c_{f}}{U_{0}}, \frac{c_{g}}{U_{0}}, \frac{U_{0}^{2}}{g} \kappa, \frac{U_{0}^{2}}{g} \beta\right)$.

For computing the group velocity, we need the relationship

$\frac{\mathrm{d} \xi}{\mathrm{d} \kappa}=\frac{c_{f}-c_{g}}{\kappa}$.

obtained by differentiating Eq. (49). The above definitions (46) and (47) for phase and group velocities remain unchanged in their dimensionless versions. We differentiate the implicit dispersion relation (48) with respect to $\kappa$ to get the formula

$2 \kappa \xi^{2}+\left(2 \kappa^{2} \xi+\beta^{2}+2 \frac{\beta}{\xi^{2}}+\frac{2}{\xi^{3}}\right) \frac{d \xi}{d \kappa}=0$,

where we insert Eq. (51). We find that the dimensionless group velocity $c_{g}$ is linked to the phase velocity as follows:

$2 \frac{\kappa^{2}}{\left(c_{f}-c_{g}\right)}\left(1-c_{f}\right)^{2}+2 \kappa^{2}\left(1-c_{f}\right)+\beta^{2}+2 \frac{\beta}{\left(1-c_{f}\right)^{2}}+\frac{2}{\left(1-c_{f}\right)^{3}}=0$.

In Table 1, we show computed values for the dimensionless phase velocity $c_{f}$ implicitly given by Eq. (48) as follows:

$\kappa^{2}\left(1-c_{f}\right)^{2}+\beta^{2}\left(1-c_{f}\right)-\beta^{2}-2 \frac{\beta}{1-c_{f}}-\frac{1}{\left(1-c_{f}\right)^{2}}=0$, 
Table 1 Phase velocity $c_{f} / U_{0}$ and group velocity $c_{g} / U_{0}$ calculated as functions of the shear rate $\beta$ and wave number $\kappa$. The upper number in each box is the dimensionless phase velocity, and the lower number is the associated group velocity. The shear rate and the wave number are given in terms of the dimensionless groups $\beta U_{0}^{2} / g$ and $\kappa U_{0}^{2} / g$.

\begin{tabular}{llccc}
\hline & $\beta U_{0}^{2} / g=0$ & $\beta U_{0}^{2} / g=0.5$ & $\beta U_{0}^{2} / g=1$ & $\beta U_{0}^{2} / g=2$ \\
\hline$\kappa U_{0}^{2} / g=0$ & & -1.875 & -1.148 & -0.682 \\
& & -1.875 & -1.148 & -0.682 \\
$\kappa U_{0}^{2} / g=0.5$ & -0.415 & -0.720 & -0.734 & -0.578 \\
& 0.293 & 0.0834 & -0.218 & -0.398 \\
$\kappa U_{0}^{2} / g=1$ & 0 & -0.208 & -0.325 & -0.380 \\
& 0.5 & 0.443 & 0.297 & 0.0159 \\
$\kappa U_{0}^{2} / g=2$ & 0.293 & 0.175 & 0.0763 & -0.0560 \\
& 0.646 & 0.633 & 0.591 & 0.447 \\
$\kappa U_{0}^{2} / g=5$ & 0.553 & 0.503 & 0.454 & 0.362 \\
& 0.776 & 0.775 & 0.769 & 0.743 \\
\hline
\end{tabular}

paired with the corresponding values for the dimensionless group velocity

$c_{g}=c_{f}+\frac{2 \kappa^{2}\left(1-c_{f}\right)^{2}}{2 \kappa^{2}\left(1-c_{f}\right)+\beta^{2}+2 \beta\left(1-c_{f}\right)^{-2}+2\left(1-c_{f}\right)^{-3}}$,

obtained by rewriting Eq. (53).

Table 1 shows dimensionless phase and group velocities in the coordinate system which is at rest with the fluid at infinite depth. Downstream wave propagation is represented by positive velocities, while upstream propagation is defined negative. We note that the long-wave domain with negative phase velocity is being extended with increasing shear rate. This trend for the phase velocity does not apply to the group velocity, which goes in the downstream direction unless the waves are both long and have a relatively strong shear.

We have now tabulated the phase and group velocity for the general problem of oscillatory waves on a exponentially varying shear flow. Apart from the case of uniform flow $(\beta=0)$, exact values for steady waves with zero phase velocity are not represented in this table. We refer to Figure 2, where the intersection points with the axis $c_{f}=0$ represent steady waves. We will now display the downstream group velocity for steady waves. It is given by the formula

$c_{g}=\frac{2 \kappa^{2}}{2 \kappa^{2}+\beta^{2}+2 \beta+2}$,

obtained by inserting $c_{f}=0$ in Eq. (55). The wave number is given by $\kappa=k$ for steady waves. Thus, we have $\kappa^{2}=1+2 \beta$ according to Eq. (33), which is inserted to provide the final formula for the group velocity of steady waves

$c_{g}=\frac{2+4 \beta}{4+6 \beta+\beta^{2}}$,

which is plotted in Fig. 3. This curve starts with the classical value 0.5 for uniform flow $(\beta=0)$, but this is not the maximal value for the group velocity. The maximum for the dimensionless group velocity of a steady wave is $c_{g}=0.5528$. It occurs for the dimensionless shear flow decay parameter $\beta=0.618$, which can be transformed back to its version with dimension by Eq. (50). The group velocity has the well-known value 0.5 in the absence of shear $(\beta=0)$ and hits this classical value once more at $\beta=2$ before decreasing to zero as $\beta^{-1}$ in the asymptotic limit $\beta \rightarrow \infty$.

In a coordinate system where the undisturbed free surface is at rest (representing uniform flow at infinite depth), a steady wave has unit phase velocity, while the dimensionless group velocity is $1-c_{g}$. From this alternative point 
Fig. 3 For a linearized steady wave, the group velocity in the downstream direction is displayed as a function of the shear flow parameters $\mathrm{U}_{0}$ and $\beta$. The dimensionless group velocity $\mathrm{c}_{\mathrm{g}} / \mathrm{U}_{0}$ is here plotted as a function of the dimensionless shear flow decay parameter $\beta \mathrm{U}_{0}^{2} / \mathrm{g}$

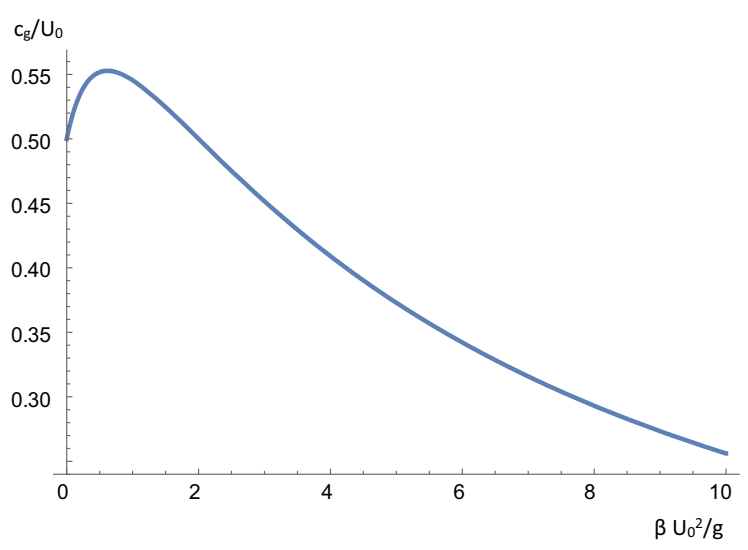

of view, the smallest ratio between group and phase velocity is 0.4472 , occurring when the dimensionless shear flow decay parameter is $\beta=0.618$. For great shear rates, the wave becomes less dispersive, as the group velocity coincides with the phase velocity in the asymptotic limit $\beta \rightarrow \infty$.

In this subsection, we have worked with dimensionless quantities, but now we go back to the original variables with dimensions.

\section{Subsurface vortex interactions}

The vortex interactions according to the vorticity equation are quadratic in the amplitude product $A_{1} A_{2}$. Each individual wave field does not generate vorticity, since there are no subsurface self-interactions of the individual normal-mode streamfunctions. We will derive the streamfunction for the induced vorticity proportional to the amplitude product $A_{1} A_{2}$. We introduce the following notations for the induced time derivative of vorticity, its underlying streamfunction, and the resulting nonlinear wave

$\dot{c}(x, y, t), \dot{\psi}(x, y, t), \quad \dot{\eta}(x, t)$,

respectively. The vorticity and streamfunction from this nonlinear subsurface interaction obey the relationship:

$\dot{c}(x, y, t)=-\nabla^{2} \dot{\psi}(x, y, t)$.

The dot represents the partial derivative with respect to time. The dot notation is reserved for the interaction wave due to quadratic subsurface vortex interactions represented by the amplitude product $A_{1} A_{2}$. The dot notation is used for distinguishing the subsurface interaction wave from the second linearized wave, which is also time dependent and provides the only oscillation in the system as long as we linearize the free-surface conditions.

The local time derivative of the induced vorticity field from the interactions between the steady wave (30)

$\psi(x, y)=A_{1} \mathrm{e}^{q y} \cos (k x-\theta)=A_{1} \mathrm{e}^{\sqrt{\beta^{2}+k^{2}} y} \cos (k x-\theta)$,

and the unsteady wave (39)

$\tilde{\psi}=A_{2} \mathrm{e}^{\alpha y} \cos (\kappa x-\omega t)$

(expressed in real variables) is found to be

$$
\begin{aligned}
\dot{c}= & A_{1} A_{2} k \alpha\left(\alpha^{2}-\kappa^{2}-\beta^{2}\right) \mathrm{e}^{(\alpha+q) y} \sin (k x-\theta) \cos (\kappa x-\omega t) \\
& +A_{1} A_{2} q \kappa\left(\beta^{2}+\kappa^{2}-\alpha^{2}\right) \mathrm{e}^{(\alpha+q) y} \cos (k x-\theta) \sin (\kappa x-\omega t),
\end{aligned}
$$

after inserting these wave components in the nonlinear vorticity equation (2). This is the induced perturbation vorticity which is of second order in the amplitude product $A_{1} A_{2}$. An individual Fourier mode does not induce selfinteraction in the vorticity equation, so there are no terms of order $A_{1}^{1}$ or $A_{2}^{2}$. We work with the original coordinate 
system where the shear flow vanishes at infinite depth. Equation (62) can be rewritten as follows:

$\dot{c}=\left(C_{1} \sin ((\kappa+k) x-\omega t-\theta)+C_{2} \sin ((\kappa-k) x-\omega t+\theta)\right) \mathrm{e}^{(\alpha+q) y}$,

introducing the amplitudes

$C_{1}=\frac{A_{1} A_{2}}{2}\left(q \kappa\left(\beta^{2}+\kappa^{2}-\alpha^{2}\right)+k \alpha\left(\alpha^{2}-\kappa^{2}-\beta^{2}\right)\right)$,

$C_{2}=\frac{A_{1} A_{2}}{2}\left(q \kappa\left(\beta^{2}+\kappa^{2}-\alpha^{2}\right)-k \alpha\left(\alpha^{2}-\kappa^{2}-\beta^{2}\right)\right)$.

The local time derivative of the associated streamfunction is

$$
\begin{aligned}
\dot{\psi}= & \left(B_{1} \sin ((\kappa+k) x-\omega t-\theta)+B_{2} \sin ((\kappa-k) x-\omega t+\theta)\right) \mathrm{e}^{(\alpha+q) y} \\
& +B_{3} \sin ((\kappa+k) x-\omega t-\theta) \mathrm{e}^{(\kappa+k) y}+B_{4} \sin ((\kappa-k) x-\omega t+\theta) \mathrm{e}^{(\kappa-k) y},
\end{aligned}
$$

where there are two known amplitudes given as follows:

$$
\begin{aligned}
& B_{1}=\frac{1}{2} \frac{A_{1} A_{2}}{(\kappa+k)^{2}+(\alpha+q)^{2}}\left(q \kappa\left(\beta^{2}+\kappa^{2}-\alpha^{2}\right)+k \alpha\left(\alpha^{2}-\kappa^{2}-\beta^{2}\right)\right), \\
& B_{2}=\frac{1}{2} \frac{A_{1} A_{2}}{(\kappa-k)^{2}+(\alpha+q)^{2}}\left(q \kappa\left(\beta^{2}+\kappa^{2}-\alpha^{2}\right)-k \alpha\left(\alpha^{2}-\kappa^{2}-\beta^{2}\right)\right) .
\end{aligned}
$$

These two rotational modes do not comprise the total flow alone, since they are unable to satisfy the free-surface conditions. For remedy, two irrotational modes had to be added, with amplitudes $B_{3}$ and $B_{4}$ which will be determined below.

Thus, there are two short waves with wave number $\kappa+k$, and two long waves with wave number $|\kappa-k|$. The two short waves have the same phase velocity, but they have different group velocities. The same is true for the two long waves. The two irrotational gravitational waves which arise in order to satisfy the linearized free-surface conditions do not contribute to the total second-order vorticity, which is still given by Eq. (62).

\subsection{The linearized wave from vortex interactions}

We will derive the induced free-surface wave from the streamfunction generated by vortex interactions. The freesurface conditions will be linearized for extracting the surface effects of subsurface interactions without confusing them with nonlinear free-surface effects. This is a legal approach to the second order in the amplitude expansion, at which the leading-order free-surface nonlinear effects can be added to the subsurface nonlinearity of vortex interactions. The linearized free-surface conditions (12) and (13) for the induced wave are differentiated in time to be written as follows:

$$
\begin{aligned}
& \frac{D \dot{\eta}}{D t}=-\left.\frac{\partial \dot{\psi}}{\partial x}\right|_{y=0}, \\
& \frac{\partial}{\partial y} \frac{D \dot{\psi}}{D t}-\beta U_{0} \frac{\partial \dot{\psi}}{\partial x}+g \frac{\partial \dot{\eta}}{\partial x}=0, \quad y=0,
\end{aligned}
$$

where we have introduced the linearized individual derivative for the surface particles

$$
\frac{D}{D t}=\frac{\partial}{\partial t}+U_{0} \frac{\partial}{\partial x} \text {. }
$$

From these kinematic and dynamic conditions, we derive a free-surface condition for the streamfunction alone

$$
\frac{\partial}{\partial y} \frac{D^{2} \dot{\psi}}{D t^{2}}-\beta U_{0} \frac{\partial}{\partial x} \frac{D \dot{\psi}}{D t}+g \frac{\partial^{2} \dot{\psi}}{\partial x^{2}}=0, \quad y=0 .
$$


Inserting the solution (66) into the condition (72) determines the amplitudes $B_{3}$ and $B_{4}$, proportional to $B_{1}$ and $B_{2}$, respectively

$$
\begin{aligned}
& \frac{B_{3}}{B_{1}}=\frac{(\beta-q-\alpha)(\kappa+k) U_{0}^{2}+(2 q+2 \alpha-\beta) \omega U_{0}-g(\kappa+k)-\omega^{2}(q+\alpha) /(\kappa+k)}{(\kappa+k-\beta)(\kappa+k) U_{0}^{2}+(\beta-2 \kappa-2 k) \omega U_{0}+g(\kappa+k)+\omega^{2}}, \\
& \frac{B_{4}}{B_{2}}=\frac{(\beta-q-\alpha)(\kappa-k) U_{0}^{2}+(2 q+2 \alpha-\beta) \omega U_{0}-g(\kappa-k)-\omega^{2}(q+\alpha) /(\kappa-k)}{(\kappa-k-\beta)(\kappa-k) U_{0}^{2}+(\beta-2 \kappa+2 k) \omega U_{0}+g(\kappa-k)+\omega^{2}}
\end{aligned}
$$

We insert the solution (66) into the kinematic condition (69) and integrate in time. Thereby, we are able to to formulate the total second-order wave which is written as the sum:

$\eta_{2}(x, t)=\eta_{\text {short }}+\eta_{\text {long }}$

given by

$$
\begin{aligned}
\eta_{\text {short }} & =-\frac{B_{1}+B_{3}}{\omega\left(\omega-k U_{0}\right)} \cos ((\kappa+k) x-\omega t-\theta), \\
\eta_{\text {long }} & =-\frac{B_{2}+B_{4}}{\omega\left(\omega-k U_{0}\right)} \cos ((\kappa-k) x-\omega t-\theta) .
\end{aligned}
$$

These waves are of second order in terms of amplitudes, through the product $A_{1} A_{2}$, as seen from Eqs. (67)-(68) upon insertion into Eqs. (73)-(74). The formulas (76)-(77) are somewhat strange because each of them seemingly represents one single wave, as far as the phase velocity is concerned. Yet, they hide two waves each, so there are in total four waves, with four different group velocities. If we consider only the surface elevation for one Fourier component, we cannot tell the difference between the short rotational wave and the short irrotational wave since they contribute symmetrically to the surface elevation by their respective amplitudes $B_{1}$ and $B_{3}$. The same relationship holds for the long rotational wave versus the long irrotational wave, having symmetric amplitude contributions $B_{2}$ and $B_{4}$.

The way to detect the underlying differences between all four waves is to trace the amplitudes $B_{1}, B_{2}, B_{3}, B_{4}$ back to formula (66). It gives the time derivative of the total streamfunction underlying the total second-order wave $\eta_{2}$. We recall that $k$ is the wavenumber of the steady-linearized wave with streamfunction amplitude $A_{1}$, with its exact dispersion relation

$k=\frac{g}{U_{0}^{2}} \sqrt{1+2 \frac{\beta U_{0}^{2}}{g}}$,

according to Eq. (33). The other wave number $\kappa$ is arbitrary (excluding the degenerate case $\kappa=k$ ), representing the added time-dependent wave with streamfunction amplitude $A_{2}$. The joint phase velocity for the short waves with wavenumber $\kappa+k$ (both rotational and irrotational) is, thus, given by

$c_{\text {short }}=\omega\left(\kappa+\frac{g}{U_{0}^{2}} \sqrt{1+2 \frac{\beta U_{0}^{2}}{g}}\right)^{-1}$,

while the similar joint phase velocity for the long waves with wavenumber $\kappa-k$ is

$c_{\text {long }}=\omega\left(\kappa-\frac{g}{U_{0}^{2}} \sqrt{1+2 \frac{\beta U_{0}^{2}}{g}}\right)^{-1}$,

where the positive direction of phase speed is in the same direction as the surface velocity $U_{0}$. This means that the short waves will always have downstream phase velocity, while the long waves will have upstream phase velocity when $\kappa<k$.

The group velocity for the rotational waves is complicated. According to Eq. (53), it is given implicitly by the equation

$2 \frac{(\kappa \pm k)^{2}}{\left(c_{f}-c_{g}\right)}\left(1-c_{f}\right)^{2}+2(\kappa \pm k)^{2}\left(1-c_{f}\right)+\beta^{2}+2 \frac{\beta}{\left(1-c_{f}\right)^{2}}+\frac{2}{\left(1-c_{f}\right)^{3}}=0$, 
where the plus sign represents $c_{f}=\omega /(\kappa+k)=c_{\text {short }}$, and the minus sign represents $c_{f}=\omega /(\kappa-k)=c_{\text {long }}$. The group velocity for irrotational waves is given explicitly as follows:

$c_{g}=\frac{U_{0}}{2}+\frac{\omega}{2}\left(\kappa \pm \frac{g}{U_{0}^{2}} \sqrt{1+2 \frac{\beta U_{0}^{2}}{g}}\right)^{-1}$,

where the plus sign represents short waves and the minus sign represents long waves. Again we emphasize that these velocities are given in the system with a surface velocity $U_{0}$.

\section{Summary and conclusions}

An analytical approach to nonlinear deep-water waves with vorticity is presented. We develop the solution in Fourier normal modes, with the wave amplitude as an expansion parameter. Our focus is the leading-order subsurface interactions between two normal-mode vortices. We represent these induced waves with a linearized free-surface condition. The present model is designed for circumventing the basic constraints of shear flow instabilities and critical layers. Thereby, we are able to extract the pure effects of subsurface vortex interactions between rotational wave modes of the basic normal-mode type. The novel effects that we have studied do not stand alone but contribute to an already established complicated picture.

The present calculations are restricted to a basic flow with an exponential variation of the velocity with height. This approach has the advantage that the steady wave that we first establish is exactly non-interacting with the basic flow, according to the vorticity equation. When we superpose two different Fourier modes as the total first-order wave, the flow becomes time dependent. Here, we use the trick of an amplitude expansion so that the second-order interaction wave can become oscillatory instead of the transient behavior to be expected from vortex interactions.

The vorticity equation requires second-order vorticity to be generated by these two rotational first-order modes in mutual interaction. However, the resulting second-order rotational flow fields cannot satisfy the free-surface conditions, even in their linearized version. Two additional irrotational waves are, therefore, produced at the free surface as compensation, preventing vorticity generation from taking place at the free surface. In total, four secondorder waves are generated by vortex interactions between two different first-order rotational modes. However, the two surface-generated irrotational modes are interwoven with their respective rotational modes, responsible for their existence. Each irrotational mode has the same phase velocity as the rotational mode behind it, and these two waves are, therefore, not distinguishable as far as the surface elevation is concerned. Analytically they can be separated because of their different group velocities, but the general formulas are complicated. We have simplified the approach by letting one of the first-order modes be steady without compromising on the fully dispersive mathematical model. With one of the two first-order modes steady, the second wave mode provides the only frequency in our model, to first order as well as second order.

Chen et al. [10] addressed finite-amplitude water waves analytically with non-uniform vorticity. They did not split the flow field into a basic flow and a wave perturbation. It is unclear whether the steady vorticity equation is satisfied by their finite-amplitude waves in light of the present work. We have demonstrated how difficult it is to avoid the generation of perturbation vorticity by interacting normal modes.

It is worth noting from the above analysis that all subsurface vortex interactions generate offspring rotational modes with a reduced vertical length scale, see Eq. (66). Only the compensatory irrotational modes generated by the free surface may induce enhanced vertical length scales. Since all wave modes fall off exponentially in the positive vertical direction, the new rotational modes will be concentrated near the surface. As such, they will play an active role in wave breaking when the wave steepness exceeds the validity of the amplitude expansion. Thereby, the waves will become transient and with reduced length scales for satisfying the vorticity equation.

We realize that wave breaking will be of a different nature with non-uniform vorticity in comparison with irrotational wave breaking. The rotational wave breaking will start at a smaller steepness while gradually developing vortex cascades with decreasing length scales just below the surface peak. The classical theory by Longuet-Higgins 
and Cokelet [23] required an excess pressure to be exerted temporarily on the surface in order to make the wave peak asymmetric for starting the breaking process. The breaking process of a nonlinear surface wave on variable shear will develop more naturally, with asymmetries arising from subsurface vortex interactions. Nonlinear vortex interactions coupled to nonlinear free-surface conditions leave a number of analytical challenges for future work.

Water waves on non-uniform flows will always be rotational. Rotational finite-amplitude waves tend to generate subsurface vortex generation. There is no self-interaction of two individual wave modes as long as each of them satisfies a Helmholtz equation. Then the vortex interactions start to second order in an amplitude expansion, with the amplitude product $A_{1} A_{2}$, where $A_{1}$ and $A_{2}$ are the first-order amplitudes of the two individual wave modes. In the present paper, we have attempted the simplest possible modeling of subsurface vortex interactions in wave modes without compromising on satisfying the exact vorticity equation to the considered order. Vortex interactions will normally induce new time dependence in the nonlinear system, but we have avoided this by letting one of the interacting waves be steady. Then the second interacting wave sets the only frequency in the system, and this will remain the only frequency also for the second-order wave of vortex interactions. This description is only possible within an amplitude expansion for the nonlinear wave, where there are two independent waves to the leading order where linearized theory is valid. Another advantage with the amplitude expansion is that we can focus exclusively on the water waves due to subsurface vorticity interactions without taking free-surface nonlinearity into account. This is because free-surface nonlinearity to the second order in the amplitudes $A_{1}$ and $A_{2}$ is independent of the subsurface interactions.

Vortex interactions will naturally be non-periodic phenomena in time, but we have designed the present model to avoid the emergence of transient behavior. This has been achieved in terms of an amplitude expansion, where the second-order wave due to vortex interactions has the same frequency as the time-dependent first-order wave. This is possible only with one steady wave among the two first-order waves.

With the simple time dependence of one single frequency in both the first-order and second-order solutions, we have focused on the higher spatial modes that are generated by subsurface vortex interactions. There are secondorder wave components as far as phase velocity is concerned, and their origin is the vortex interactions between the two rotational modes in the first-order solution. The wave numbers of these components are either the sum or the difference of the wave numbers for each of the two given first-order modes. However, there are four distinct physical waves which become apparent when we consider the group velocities. Each of the two rotational waves will induce its offspring irrotational wave at the free surface in order to avoid that vorticity is generated at the free surface. Each rotational wave and its accompanying irrotational wave will have different group velocities. This will lead to escalating nonlinear interactions, where the irrotational modes tear the rotational modes apart and generate new rotational modes with smaller length scales. These cumulative transient nonlinear interactions between the rotational modes and their induced irrotational modes will be important in wave breaking but are left as a challenge for later work.

Funding Open access funding provided by Norwegian University of Life Sciences

Open Access This article is licensed under a Creative Commons Attribution 4.0 International License, which permits use, sharing, adaptation, distribution and reproduction in any medium or format, as long as you give appropriate credit to the original author(s) and the source, provide a link to the Creative Commons licence, and indicate if changes were made. The images or other third party material in this article are included in the article's Creative Commons licence, unless indicated otherwise in a credit line to the material. If material is not included in the article's Creative Commons licence and your intended use is not permitted by statutory regulation or exceeds the permitted use, you will need to obtain permission directly from the copyright holder. To view a copy of this licence, visit http://creativecommons.org/licenses/by/4.0/.

\section{References}

1. Simmen JA, Saffman PG (1985) Steady deep-water waves on a linear shear current. Stud Appl Math 73:35-57

2. Teles da Silva AF, Peregrine DH (1988) Steep, steady surface waves on water of finite depth with constant vorticity. J Fluid Mech 195:281-302

3. Hur VM, Wheeler MH (2020) Exact free surfaces in constant vorticity flows. J Fluid Mech 896:R1 
4. Dalrymple RA (1977) A numerical model for periodic finite amplitude waves on a rotational fluid. J Comput Phys 24:29-44

5. Gerstner FJ (1802) Theorie der Wellen. Abh König Böhm Ges Wiss (Reprinted in: Ann Phys 32, 412-445, 1809)

6. Lamb H (1932) Hydrodynamics. Cambridge University Press, Cambridge

7. Nwogu OG (2009) Interaction of finite-amplitude waves with vertically sheared current fields. J Fluid Mech 627:179-213

8. Constantin A, Sattinger D, Strauss W (2006) Variational formulations for steady water waves with vorticity. J Fluid Mech 548:151163

9. Ko J, Strauss W (2008) Effect of vorticity on steady water waves. J Fluid Mech 608:197-215

10. Cheng J, Cang J, Liao S-J (2009) On the interaction of deep water waves and exponential shear currents. Z Angew Math Phys 60:450-478

11. Milewski PA, Benney DJ (1995) Resonant interactions between vortical flows and water waves. Part I: deep water. Stud Appl Math 94:131-167

12. Peregrine DH (1976) Interaction of water waves and currents. Adv Appl Mech 16:9-117

13. Sarpkaya T (1996) Vorticity, free surface, and surfactants. Ann Rev Fluid Mech 28:83-128

14. Telste JG (1989) Potential flow about two counter-rotating vortices approaching a free surface. J Fluid Mech 201:259-278

15. Tyvand PA (1991) Motion of a vortex near a free surface. J Fluid Mech 225:673-686 (Appendix by R.P. Tong)

16. Wu GX (1994) Wave radiation by a submerged source undergoing large amplitude periodic motion. J Eng Math 28:447-461

17. Sarpkaya T, Isaachson M (1981) Mechanics of wave forces on offshore structures. Van Nostrand Reinhold, New York

18. Tyvand PA (2020) Initial stage of the finite-amplitude Cauchy-Poisson problem. Water Waves 2:145-169

19. Miles JW (1962) On the generation of surface waves by shear flow. Part 4. J Fluid Mech 13:433-448

20. Drazin PG, Reid WH (1981) Hydrodynamic stability. Cambridge University Press, Cambridge

21. Craik ADD (2011) Wave interactions and fluid flows. Cambridge University Press, Cambridge

22. Brevik I, Sollie R (1993) Energy flux and group velocity in currents of uniform vorticity. Quart J Mech Appl Math 46:117-130

23. Longuet-Higgins MS, Cokelet ED (1976) The deformation of steep surface waves on water-I. A numerical method of computation. Proc R Soc A 350:1-16

Publisher's Note Springer Nature remains neutral with regard to jurisdictional claims in published maps and institutional affiliations. 